\title{
Diagnostic challenges in cervical tuberculous lymphadenitis: A review
}

\author{
Hande Senem Deveci, ${ }^{1}$ Mustafa Kule, ${ }^{2}$ Zeynep Altin Kule, ${ }^{2}$ Tulay Erden Habesoglu ${ }^{1}$ \\ ${ }^{1}$ Departmen of Ear, Nose and Throat, Fatih Sultan Mehmet Training and Research Hospital, Istanbul, Turkey \\ ${ }^{2}$ Departmen of Ear, Nose and Throat, Mugla State Hospital, Mugla, Turkey
}

\begin{abstract}
Tuberculosis is a very serious disease and incidence is once again on the rise. Lymph node tuberculosis is one of the most common extrapulmonary manifestations of tuberculosis. In differential diagnosis of chronic, painless cervical lymphadenopathy, cervical tuberculous lymphadenitis should be kept in mind. A high index of suspicion is needed for diagnosis of tuberculous lymphadenitis, which is known to mimic a number of pathological conditions. This article reviews epidemiology, clinical manifestations, and diagnostic techniques for cervical tuberculous lymphadenitis.

Keywords: Cervical tuberculous lymphadenitis; extrapulmonary tuberculosis; tuberculosis.
\end{abstract}

$\mathrm{T}$ uberculosis $(\mathrm{TB})$ is a common, and in many cases lethal, infectious disease caused by various strains of mycobacteria, usually Mycobacterium tuberculosis [1]. Today in developing countries tuberculosis is still a major health problem. As a consequence of increased human immunodeficiency virus (HIV) prevalence and increasing immigration rate, tuberculosis (TB) is also re-emerging as a health care problem in developed countries [2]. Tuberculosis which mainly involves the lungs can also cause infection in almost all other organs and tissues in the body. TB bacilli enter the lymphatic system and blood stream to reach the extrapulmonary organs. Notable extrapulmonary infection sites include the pleura, the central nervous system, the lymphatic system, the genitourinary system, and the bones and joints [3]. Lymph node tuberculosis (LNT) is a common cause of lymphadenopathy in areas in which TB is endemic. In countries with a low prevalence of TB, LNT is the most common extrapulmonary form [4]. In contrast, in high prevalence areas, the LNT incidence is second to that of TB pleuritis [2]. The most common LNT form is mycobacterial cervical lymphadenitis (MCL) [5, 6]. Although new diagnostic methods have been developed, especially in patients without a history of tuberculosis, the cervical tuberculous lymphadenitis (CTL) diagnosis is still elusive. In differential diagnosis of CTL, other granulomatous lymphadenitis should be considered such as non-tuberculous mycobacteria (including M. scrofulaceum, M. avium, and M. baemophilum), sarcoidosis, toxoplasmosis, tularemia, fungal disease, cat-scratch disease and neoplasms $[7,8]$. The diagnosis is necessitating a high index of suspicion.

Received: January 25, 2016 Accepted: June 23, 2016

Correspondence: Dr. Hande Senem DEVECI. Fatih Sultan Mehmet Egitim ve Arastirma Hastanesi, Kulak Burun Bogaz Klinigi, Istanbul, Turkey. 
Kent and et.al. alleged that CTL is the result of lympho-hematogenous spread of pulmonary tuberculosis [9]. According to Powell, this entity is a hyperreaction of lymph nodes against previous pulmonary tuberculosis [10]. And also Yew et al. suggested that the predominant pathway of spread of the tubercle bacilli to the cervical lymph nodes is from lung parenchyma as the lymphatics of the right lung and the lower lobe of the left lung normally drain to the right supraclavicular lymph nodes and then upwards to the right lower cervical chain [11]. However, the pathogenesis of CTL without pulmonary tuberculosis cannot be explained by this theory, and also alternate routes of spread to lymph nodes, such as the tonsils and adenoids, have been proposed [12]. Lymph node tuberculosis could be also occurring by direct exposure to infection [3].

In this review, we aimed to discuss the diagnostic methods of CTL and to evaluate the usefulness of these diagnostic methods.

\section{Dermographic findings}

Age and gender distribution of CTL is different from the pulmonary tuberculosis. CTL is more frequent in females and in the younger age groups, whereas pulmonary tuberculosis is more common in males and in the older age groups $[2,13,14]$. Dandapat et al. have suggested that this phenomenon occurs as a consequence of male-dominated communities, where women experience poorer living conditions, and because young females generally notice differences in their appearance earlier than males [15].

\section{Clinical presentation}

In the differential diagnosis of a cervical mass, CTL should be kept in mind especially in endemic areas. CTL may present as a unilateral single or multiple painless lump, mostly located in the posterior cervical or supraclavicular region $[6,16]$. The duration of lymphadenopathy at time of presentation is typically 1-2 months, varying from 3 weeks to 8 months $[8,17]$. Fistula formation can be seen in almost $10 \%$ of the mycobacterial cervical lymphadenitis $[6,18]$, though it is rare in atypical cases [19]. There is a significant variability in the literature on the occurrence of clinical signs and symptoms of LNT other than the cervical mass. However night sweats, weakness, cough and fever could be also seen in these patients in different ratios $[2,15]$. These systemic symptoms are more commonly seen in HIV positive patients [20].

Also in HIV-positive patients the lymphadenopathies are more commonly seen as symmetrical and multiple contrasted with presentation with focal and asymmetrical lymphadenopathies of HIVnegative patients [21].

Accompanying pulmonary tuberculosis is reported in $18 \%-42 \%$ of patients. The higher rate of pulmonary tuberculosis is seen in HIV-positive patients rather than HIV-negative patients [22].

\section{Diagnostic tools}

In order to make a diagnosis of CTL suspicion is mandatory. A detailed history and physical examination which is supported with hematological tests, tuberculin test, imaging techniques, fine-needle aspiration (FNA), and molecular tests will help arrive at an early diagnosis of tuberculous lymphadenitis and allow early initiation of treatment before the final diagnosis can be made by incisional biopsy and culture $[8,23,24]$.

\section{Hematological tests}

Although there is no specific blood test, leukocytosis, thrombocytosis, anemia, hyponatremia and increased ALP results are associated with chronic disease condition and these results create a doubt about an infective condition. Also elevated ESR show up a non-specific inflammatory response $[25,26]$.

Extrapulmonary involvement can be seen in more than $50 \%$ of patients with concurrent AIDS and pulmonary tuberculosis [3]. Because of the coinfection risk of extrapulmonary forms of tuberculosis in HIV-positive patients, all patients with CTL suspects have to be tested for HIV [14, 26, 27].

\section{Tuberculin skin test}

Tuberculin skin test (TST) is used to show delayed-type hypersensitivity reactions against mycobacterial antigen, in which the reagent is mostly protein purified derivative (PPD). The test becomes positive 2-10 weeks after the mycobacterial infection. Positive reactions ( $>10-\mathrm{mm}$ induration) can occur in M. tuberculosis infections. Suspicious reac- 
tions (5- to 9-mm induration) can occur after BCG vaccination, $M$. tuberculosis infection or nontuberculous mycobacterial infections. Negative reactions $(<4-\mathrm{mm}$ induration) represent a lack of tuberculin sensitization. False-negative reactions can occur in at least $20 \%$ of all people with active tuberculosis. The test may also be false positive in different conditions, like other infections, metabolic disease, malnutrition, live virus vaccination, malignancy, immunosuppressive drugs, newborns, elderly people, stress, sarcoidosis and inadequate test application [28]. Mandatory Bacille Calmette-Guerin (BCG) vaccination is in some countries may increase the false-positivity [2].

Although the value of the test is controversial [29], the positivity of TST is up to $98 \%$ in USA [17] and $100 \%$ in Turkey [30] in HIV-negative tuberculosis patients. Thus TST is a valuable, but nonspecific test for assessment of TB in Turkey $[2,30]$.

\section{Imaging techniques}

Chest X-ray, neck ultrasound (USG), computerized tomography (CT) and magnetic resonance imaging (MRI) of neck can be performed in cervical lymphadenopathies.

Chest X-ray findings may be positive in $10-40 \%$ of patients [31]. However, the normal chest X-ray should not exclude the CTL diagnosis.

The changes in size, shape (L/T ratio), echogenicity and morphology of the nodal hilum or cortex have been suggested as sonographic criteria for differentiation of benign pathologies from malignant. However, USG findings do not show diseasespecific features for CTL. It can also be used as an imaging tool for the guided aspirations. Therefore, when combined with fine-needle aspiration, it has a very high sensitivity and specificity [32].

CT and MRI are valuable complementary techniques in evaluation of CTL. However, they are not sufficient to make a certain diagnosis. They accurately demonstrate the sites, pattern and extend of the disease. Imaging features are varied and nonspecific, although rim enhancement or calcification, if present, can be a strong indicator of the disease [33]. There are three patterns of nodal involvement in tuberculosis lymphadenitis on CT or MR images $[34,35]$. In the early course of disease, the nodes are homogenous in attenuation and after adminis- tration of intravenous contrast they enhance homogeneously. As the disease progress, the second and the most common pattern, a node with central area of necrosis, is seen. At CT, the affected nodes have center of low attenuation with an enhancing rim. At MRI, the enhancing areas are of intermediate signal intensity with $\mathrm{T} 1$-weighted sequences and are hypointense with T2-weighted sequences, whereas non-enhancing areas are relatively hypointense with T1-weighted sequences and markedly hyperintense with T2-weighted sequences. The non-enhancing areas indicate caseation or liquefaction necrosis, and the enhancing areas indicate granulation tissue with an inflammatory hypervascularity and increased vascular permeability. The third pattern is a fibrocalcified node that is usually seen in patients who have been treated. At CT, the node is homogenous and the calcification could be noticed. At MRI, it is homogenously hypointense both in T1- and T2weighted sequences and does not enhance after injection of contrast material $[33,34]$.

\section{Fine-needle aspiration}

In fine-needle aspiration (FNA), a thin needle is inserted into an infected, swollen, superficial lymph node. Then, the taken aspirate material could be allocated for cytological examination, acid-fast bacilli (AFB) staining, culturing and/or molecular testing.

Fine-needle aspiration cytology shows up a wellformed epithelioid granuloma and the presence of caseous necrosis [36]. These finding are highly suggestive of tubercular etiology, especially in developing countries where the incidence of tuberculosis is high [36]. The sensitivity and specificity of FNA cytology in the diagnosis of tuberculous lymphadenitis are $88 \%$ and $96 \%$, respectively [37]. However, typical granulomas and caseation are less likely to be found in HIV-positive tuberculosis lymphadenitis because of the impaired T-cell function [20]. Therefore bacteriological confirmation is essential. Combination of FNA cytology with the culture or a TST further increases the diagnostic yield in CTL $[29,38]$.

FNA material also subjected to Ziehl-Neelsen $(\mathrm{ZN})$ staining for AFB, mycobacterial culture and molecular test [20].

$\mathrm{ZN}$ staining and microscopic evaluation is rapid, cheap and easy. Sensitivity ranges from $46-78 \%$ 
and the specificity is actually $100 \%$ [36]. But the sensitivity ratio varies according to the source of the sample. Tandesse et al, revealed that concentrated aspirate material increase the yield of AFB staining procedure [39].

\section{Culture}

A definitive diagnosis of tuberculosis lymphadenitis can be made by demonstration of $M$. tuberculosis in an affected lymph node by culture. However, a negative culture result should not exclude the diagnosis of CTL [28]. Isolation of mycobacterium by culture is possible in $10-69 \%$ of the cases $[6,24,40]$. And also the long duration of culture (6-8 weeks) cause delay in initiation of treatment and is assessed as time-consuming.

\section{Molecular testing}

Polymerase chain reaction (PCR) which is a nucleic acid amplification test, provide a rapid, specific and sensitive diagnosis of M. tuberculosis. [24]. After FNAs, PCR should be performed for the samples. When we scan the literature, the positivity of PCR from FNA materials were found $71.4 \%, 76.4 \%$ and $92.1 \%$ in three different studies $[41,42]$. In one of these studies, Suzuki K. et al. compared the sensitivity of cytological examination, smears, cultures and PCR technique using an aspiration procedure for cervical tuberculous lymphadenitis. And the ratios of these techniques were $13.3 \%, 50 \%, 60 \%$ and $71.4 \%$, respectively [43].

Histopathologic examination and PCR of an excisional biopsy should be recommended only for patients in whom FNA-PCR is negative or when there is discrepancy with the clinical impression [24].

\section{Histopathology}

Histopathologic examination is one of the most important diagnostic method of CTL $[6,44,45$, 46]. Langerhans giant cells, caseating necrosis, granulomatous inflammation and calcification can be seen in histopathological examination [47]. Though histopathology is most reliable method for diagnosis of cervical lymphadenitis, its feasibility is limited due to its non-acceptability, as it is an invasive procedure [20]. Although it's an invasive technique, early surgical excision of the tubeculous lymphadenitis is considered as an adjunct to antibiotic therapy. So surgical excision has been recommended for treatment failure cases of tuberculosis lymphadenitis and for patients who have discomfort from tense, fluctuant lymph nodes $[17,31]$. Preferred surgical method is excision of an infected lymph node. Because incisional biopsy is associated with sinus tract and fistula formation and therefor is contraindicated [48].

\section{Conclusion}

Tuberculosis (TB) is a major health concern in developing countries. This disease is a systemic disease which may give rise to cervical lymphadenitis as an extrapulmonary manifestation of the disease. The most usual signs and symptoms are the appearance of a chronic, painless mass in the neck, which is persistent and usually grows with time. Because of no other remarkable symptom their diagnosis and distinction need a high index of suspicion, and application of a variety of diagnostic modalities. However, it is not possible or practical to apply all of the diagnostic procedures in all patients. This would be time consuming and expensive. Increased ESR, leukocytosis thrombocytosis, anemia, hyponatremia and increased ALP results put forward a non-specific inflammation or a chronic disease status. Chest radiographs do not have any diagnostic value. And tuberculin skin test is also not valuable in areas where BCG vaccination is mandatory. CT and MRI scans may show characteristic signs and the localization of the CTL which may help if the surgical excision would be planned in future. After a FNA, a combination of conventional techniques (such as cytology, staining, culture) with PCR must be applied for the rapid and early diagnosis of CTL. Therefore, the value of FNA is indisputable. The definitive diagnosis of tuberculous lymphadenitis is done by excisional biopsy and histopathologic examination if all other techniques fail.

\section{Conflict of Interest: None declared.}

Financial Disclosure: The authors declared that this study has received no financial support.

\section{REFERENCES}

1. Kumar V, Abbas AK, Fausto N, Mitchell RN. Robbins Basic Pathol- 
ogy (8th ed.). Saunders Elsevier 2007. p. 516-22.

2. Tatar D, Senol G, Alptekin S, Gunes E. Assessment of lymph node tuberculosis in two provinces in Turkey. Jpn J Infect Dis 2011;64:316-21.

3. Golden MP, Vikram HR. Extrapulmonary tuberculosis: an overview. Am Fam Physician 2005;72:1761-8.

4. Centers for disease control and prevention. reported tuberculosis in the united states, 2005. Available at: http://www.cdc.gov/tb/statistics/reports/surv2005/default.htm.

5. Taşbakan MS, Pullukçu H, Sipahi OR, Işıkgöz Taşbakan M, Ozkören Çalık S, Yamazhan T. Evaluation of 694 tuberculous lymphadenitis cases reported from Turkey between 1997-2009 period by pooled analysis method. Mikrobiyol Bul 2010;44:385-93.

6. Kanlikama M, Mumbuç S, Bayazit Y, Sirikçi A. Management strategy of mycobacterial cervical lymphadenitis. J Laryngol Otol 2000;114:274-8. Crossref

7. Asano S. Granulomatous lymphadenitis. J Clin Exp Hematop 2012;52:1-16. Crossref

8. Artenstein AW, Kim JH, Williams WJ, Chung RC. Isolated peripheral tuberculous lymphadenitis in adults: current clinical and diagnostic issues. Clin Infect Dis 1995;20:876-82. Crossref

9. Kent DC. Tuberculous lymphadenitis: not a localized disease process. Am J Med Sci 1967;254:866-74. Crossref

10. Powell DA. Tuberculous lymphadenitis. In: Schlossberg D, ed. Tuberculosis and nontuberculous mycobacterial infections. 4th ed. Philadelphia: WB Saunders Company 1999:186-94.

11. Yew WW, Lee J. Pathogenesis of cervical tuberculous lymphadenitis: pathways to anatomic localization. Tuber Lung Dis 1995;76:275-6.

12. Selimoğlu E, Sütbeyaz Y, Ciftçioğlu MA, Parlak M, Esrefoğlu M, Oztürk A. Primary tonsillar tuberculosis: a case report. J Laryngol Otol 1995;109:880-2. Crossref

13. Jha BC, Dass A, Nagarkar NM, Gupta R, Singhal S. Cervical tuberculous lymphadenopathy: changing clinical pattern and concepts in management. Postgrad Med J 2001;77:185-7. Crossref

14. Geldmacher H, Taube C, Kroeger C, Magnussen H, Kirsten DK. Assessment of lymph node tuberculosis in northern Germany: a clinical review. Chest 2002;121:1177-82. Crossref

15. Dandapat MC, Mishra BM, Dash SP, Kar PK. Peripheral lymph node tuberculosis: a review of 80 cases. Br J Surg 1990;77:911-2.

16. Penfold CN, Revington PJ. A review of 23 patients with tuberculosis of the head and neck. Br J Oral Maxillofac Surg 1996;34:508-10.

17. Polesky A, Grove W, Bhatia G. Peripheral tuberculous lymphadenitis: epidemiology, diagnosis, treatment, and outcome. Medicine (Baltimore) 2005;84:350-62. Crossref

18. Konishi K, Yamane H, Iguchi H, Nakagawa T, Shibata S, Takayama $\mathrm{M}$, et al. Study of tuberculosis in the field of otorhinolaryngology in the past 10 years. Acta Otolaryngol Suppl 1998;598:244-9.

19. Olson NR. Atypical mycobacterial cervical lymphadenitis: Clinical presentation. Laryngoscope 1967;77:1376-9. Crossref

20. Handa U, Mundi I, Mohan S. Nodal tuberculosis revisited: a review. J Infect Dev Ctries 2012;6:6-12. Crossref

21. Bem C. Human immunodeficiency virus-positive tuberculous lymphadenitis in Central Africa: clinical presentation of 157 cases. Int J Tuberc Lung Dis 1997;1:215-9.

22. Shriner KA, Mathisen GE, Goetz MB. Comparison of mycobacteri- al lymphadenitis among persons infected with human immunodeficiency virus and seronegative controls. Clin Infect Dis 1992;15:6015. Crossref

23. Ibekwe AO, Shareef Z, Kindy S. Diagnostic problems of tuberculous cervical adenitis (scrofula). Am J Otolaryngol 1997;18:202-5.

24. Singh KK, Muralidhar M, Kumar A, Chattopadhyaya TK, Kapila $\mathrm{K}$, Singh MK, et al. Comparison of in house polymerase chain reaction with conventional techniques for the detection of Mycobacterium tuberculosis DNA in granulomatous lymphadenopathy. J Clin Pathol 2000;53:355-61. Crossref

25. Fitzpatrick EL, LeJeune FE Jr. Mycobacterial cervical lymphadenitis: a review. J La State Med Soc 1996;148:451-4.

26. Yoon HJ, Song YG, Park WI, Choi JP, Chang KH, Kim JM. Clinical manifestations and diagnosis of extrapulmonary tuberculosis. Yonsei Med J 2004;45:453-61. Crossref

27. Barnes PF, Lakey DL, Burman WJ. Tuberculosis in patients with HIV infection. Infect Dis Clin North Am 2002;16:107-26. Crossref

28. Bayazit YA, Bayazit N, Namiduru M. Mycobacterial cervical lymphadenitis. ORL J Otorhinolaryngol Relat Spec 2004;66:275-80.

29. Lau SK, Wei WI, Kwan S, Yew WW. Combined use of fine-needle aspiration cytologic examination and tuberculin skin test in the diagnosis of cervical tuberculous lymphadenitis. A prospective study. Arch Otolaryngol Head Neck Surg 1991;117:87-90. Crossref

30. Cinar F, Cinar S, Yilmaz B, Gürsel O. Purified protein derivative: the vital part of the cervical tuberculous adenitis diagnosis. Otolaryngol Head Neck Surg 2003;129:245-7. Crossref

31. Fontanilla JM, Barnes A, von Reyn CF. Current diagnosis and management of peripheral tuberculous lymphadenitis. Clin Infect Dis 2011;53:555-62. Crossref

32. Gupta A, Rahman K, Shahid M, Kumar A, Qaseem SM, Hassan SA, et al. Sonographic assessment of cervical lymphadenopathy: role of high-resolution and color Doppler imaging. Head Neck 2011;33:297-302.

33. Moon WK, Han MH, Chang KH, Im JG, Kim HJ, Sung KJ, et al. $\mathrm{CT}$ and MR imaging of head and neck tuberculosis. Radiographics 1997;17:391-402. Crossref

34. Moon WK, Im JG, Yu IK, Lee SK, Yeon KM, Han MC. Mediastinal tuberculous lymphadenitis: MR imaging appearance with clinicopathologic correlation. AJR Am J Roentgenol 1996;166:21-5.

35. Lee Y, Park KS, Chung SY. Cervical tuberculous lymphadenitis: CT findings. J Comput Assist Tomogr 1994;18:370-5. Crossref

36. Mittal P, Handa U, Mohan H, Gupta V. Comparative evaluation of fine needle aspiration cytology, culture, and PCR in diagnosis of tuberculous lymphadenitis. Diagn Cytopathol 2011;39:822-6. Crossref

37. Gupta SK, Chugh TD, Sheikh ZA, al-Rubah NA. Cytodiagnosis of tuberculous lymphadenitis. A correlative study with microbiologic examination. Acta Cytol 1993;37:329-32.

38. Ellison E, Lapuerta P, Martin SE. Fine needle aspiration diagnosis of mycobacterial lymphadenitis. Sensitivity and predictive value in the United States. Acta Cytol 1999;43:153-7. Crossref

39. Tadesse M, Abebe G, Abdissa K, Bekele A, Bezabih M, Apers L, et al. Concentration of lymph node aspirate improves the sensitivity of acid fast smear microscopy for the diagnosis of tuberculous lymphadenitis in Jimma, southwest Ethiopia. PLoS One 2014;9:106726.

40. Kanlikama M, Ozsahinoglu C, Akan E, Ozcan K. Mycobacterial 
species causing cervicofacial infection in Turkey. Eur Arch Otorhinolaryngol 1993;250:237-9. Crossref

41. Baek CH, Kim SI, Ko YH, Chu KC. Polymerase chain reaction detection of Mycobacterium tuberculosis from fine-needle aspirate for the diagnosis of cervical tuberculous lymphadenitis. Laryngoscope 2000;110:30-4. Crossref

42. Chiesa Estomba CM, Betances Reinoso FA, Rivera Schmitz T, Ossa Echeverri CC, González Cortés MJ, Santidrian Hidalgo C. Head and neck tuberculosis: 6-year retrospective study. Acta Otorrinolaringol Esp. 2016;67:9-14. Crossref

43. Suzuki K, Yagi M, Sakagami T, Fujisawa T, Miyamoto M, Kobayashi Y, et al. A Clinical Study on Cervical Tuberculous Lymphadenitis. [Article in Japanese] Nihon Jibiinkoka Gakkai Kaiho 2015;118:643-50. [Abstract] Crossref
44. Alleva M, Guida RA, Romo T 3rd, Kimmelman CP. Mycobacterial cervical lymphadenitis: a persistent diagnostic problem. Laryngoscope 1988;98:855-7. Crossref

45. Manolidis S, Frenkiel S, Yoskovitch A, Black M. Mycobacterial infections of the head and neck. Otolaryngol Head Neck Surg 1993;109:427-33. Crossref

46. Lee KC, Tami TA, Lalwani AK, Schecter G. Contemporary management of cervical tuberculosis. Laryngoscope 1992;102:60-4.

47. Suskind DL, Handler SD, Tom LW, Potsic WP, Wetmore RF. Nontuberculous mycobacterial cervical adenitis. Clin Pediatr (Phila) 1997;36:403-9. Crossref

48. Cantrell RW, Jensen JH, Reid D. Diagnosis and management of tuberculous cervical adenitis. Arch Otolaryngol 1975;101:53-7. 